\title{
Surgical correction of the severe jaw deformity in teenagers with juvenile idiopathic arthritis
}

\author{
Larry M. Wolford \\ Departments of Oral and Maxillofacial Surgery and Orthodontics, Texas A\&M University College of Dentistry, Baylor University \\ Medical Center, Dallas, TX 75246, USA. \\ Correspondence to: Prof. Larry M. Wolford, Departments of Oral and Maxillofacial Surgery and Orthodontics, Texas A\&M \\ University College of Dentistry, Baylor University Medical Center, 3409 Worth St. Suite \#400, Dallas, TX 75246, USA. E-mail: \\ Iwolford@drlarrywolford.com
}

How to cite this article: Wolford LM. Surgical correction of the severe jaw deformity in teenagers with juvenile idiopathic arthritis. Plast Aesthet Res 2021;8:31. https://dx.doi.org/10.20517/2347-9264.2021.12

Received: 5 Feb 2021 First Decision: 31 Mar 2021 Revised: 10 Apr 2021 Accepted: 30 Apr 2021 Available online: 19 Jun 2021

Academic Editor: Pedro Infante-Cossio Copy Editor: Xi-Jun Chen Production Editor: Xi-Jun Chen

\begin{abstract}
Juvenile idiopathic arthritis (JIA) can create severe jaw deformities affecting function, esthetics and psychosocial health of teenagers afflicted with this disease. The aim of this chapter is to present the dentofacial manifestations of this disease and the proven surgical protocol to correct these debilitating deformities. Clinical and imaging characteristics of JIA patients with temporomandibular joint (TMJ) involvement and severe jaw deformities are presented as well as the surgical protocol to produce predictable stable outcomes. Clinical research studies documenting the efficacy of this surgical protocol will be reviewed. The most predictable, successful, and stable surgical protocol to treat JIA patients with severe dentofacial deformities includes: bilateral TMJ reconstruction and mandibular counterclockwise rotation-advancement with patient-fitted TMJ total joint prostheses (TJP) and concomitant maxillary osteotomies, as well as adjunctive procedures, performed in a single operation. This protocol provides improvement in jaw function, facial esthetics, pain, and airway. Research studies document the predictability of the treatment protocol. Case presentations illustrate the deformity and the expected outcomes with this surgical protocol. Patient-fitted TJP for TMJ and mandibular reconstruction in conjunction with maxillary orthognathic surgery provides long-term skeletal and occlusal stability in the JIA patient as well as improvement in jaw function, pain, esthetics and airway.
\end{abstract}


Keywords: Juvenile idiopathic arthritis, temporomandibular joint, oropharyngeal airway, sleep apnea, temporomandibular joint concepts, temporomandibular joint total joint prosthesis, condylar resorption

\section{INTRODUCTION}

Juvenile idiopathic arthritis (JIA) is an auto-immune condition and is the most common chronic joint disease in childhood. It affects up to 150 in 100,000 children worldwide ${ }^{[1]}$. There are seven basic categories of JIA, including: (1) systemic onset; (2) oligoarthritis ( $\leq 4$ joints); (3) rheumatoid factor positive polyarthritis ( $\geq 5$ joints); (4) rheumatoid factor negative polyarthritis ( $\geq 5$ joints); (5) psoriatic arthritis; (6) enthesitis; and (7) undifferentiated arthritis ${ }^{[2]}$. The onset of JIA is in the first or second decade (16-year-old or younger), with greater occurrence in females (59\%-78\%). The triggers and precise pathophysiology are unknown. Multiple systems are usually involved. Joint damage may be mediated by cytokines, chemokines, and metalloproteases. Peripheral joints are usually symmetrically inflamed resulting in progressive destruction of articular structures, commonly accompanied with systemic symptoms. JIA can be a monoarticular or commonly a polyarticular disease process. TMJ involvement can create significant functional, aesthetic, and painful jaw deformities. The destructive temporomandibular joint (TMJ) pathology in children with chronic arthritis was first reported by Still ${ }^{[3]}$ in 1897 where an associated significant jaw deformity was attributed to the destructive process in the TMJs.

The reported involvement of the TMJs in the JIA disease process ranges from $25 \%$ to $70 \%{ }^{[1]}$, and may be asymptomatic or painful and dysfunctional. Significant condylar resorption can occur with development of a major jaw deformity. Unlike other synovial joints, the mandibular growth plate lies directly beneath a thin layer of fibrocartilage at the surface of the condyle and is the primary growth center of the mandibular condyle that extends from the prenatal period until completion of pubertal growth. Damage to this area interrupts mandibular growth creating the jaw deformity [Figure 1A-C, Figure 2A-C, Figure 3A]. Patients may have pain, headache, malocclusion, difficulties eating and chewing, micrognathia, decreased oropharyngeal airway, and sleep apnea [Figure 3A]. Children with JIA have a higher occurrence rate of TMJ pain, headaches, neck pain, and jaw dysfunction than healthy controls at time of diagnosis and at long-term follow-up ${ }^{[4]}$. JIA patients with active or end-stage TMJ involvement can present distinct clinical and imaging characteristics, with the degree of affect influenced by age of onset (younger onset, worse the deformity), disease aggression, length of time since onset and effect of interceptive medicine.

\section{Clinical and imaging features}

JIA characteristics may include: (1) progressive retrusion of the mandible with worsening skeletal and occlusal deformity [Figure 1A-C, Figure 2A-C]; (2) high occlusal plane angle facial morphology [Figure 1C, Figure 3B]; (3) maxillary anteroposterior (AP) and posterior vertical hypoplasia, but can also have anterior vertical hyperplasia; (4) Class II skeletal and occlusal relation with or without anterior open bite; (5) condylar and articular eminence resorption [Figure 4]; (6) MRI evidence of bone resorption and pannus surrounding the degenerative disc [Figure 5]; (7) TMJs can be asymptomatic, but may elicit clicking, crepitus, TMJ dysfunction, TMJ pain, headache, myofascial pain, earache, tinnitus, vertigo, and so on; (8) decreased maximum incisal opening (MIO); (9) other joints and systems commonly involved; and (10) airway obstruction related to hyperplastic inferior turbinates, deviated nasal septum, and decreased oropharyngeal airway, which can result in sleep apnea.

Teenage JIA patients with some or all of these characteristics may benefit from TMJ reconstruction with patient-fitted TMJ Concepts (TMJ Concepts Inc., Ventura CA) total joint prosthesis (TJP) [Figure 6] and concomitant orthognathic surgery ${ }^{[5-15]}$. The purpose of this chapter is to demonstrate the single-stage 

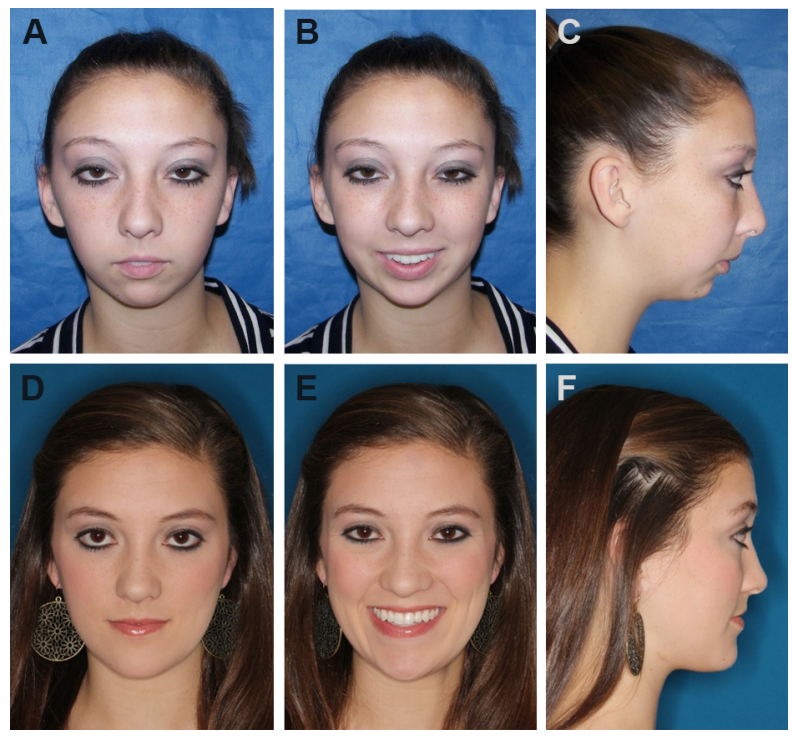

Figure 1. Case 1: 16-year-old female with JIA affecting the TMJs with severe condylar resorption resulting in a significant dentofacial deformity. Frontal view (A). Smiling view (B). Profile view (C). The patient is seen 12 months post-surgery following TJP-Max-SP and genioplasty. Frontal view (D). Smiling view (E). Profile view (F). JIA: Juvenile idiopathic arthritis; TMJs: temporomandibular joints; TJPMax-SP: total joint prostheses with maxillary osteotomies surgical protocol.
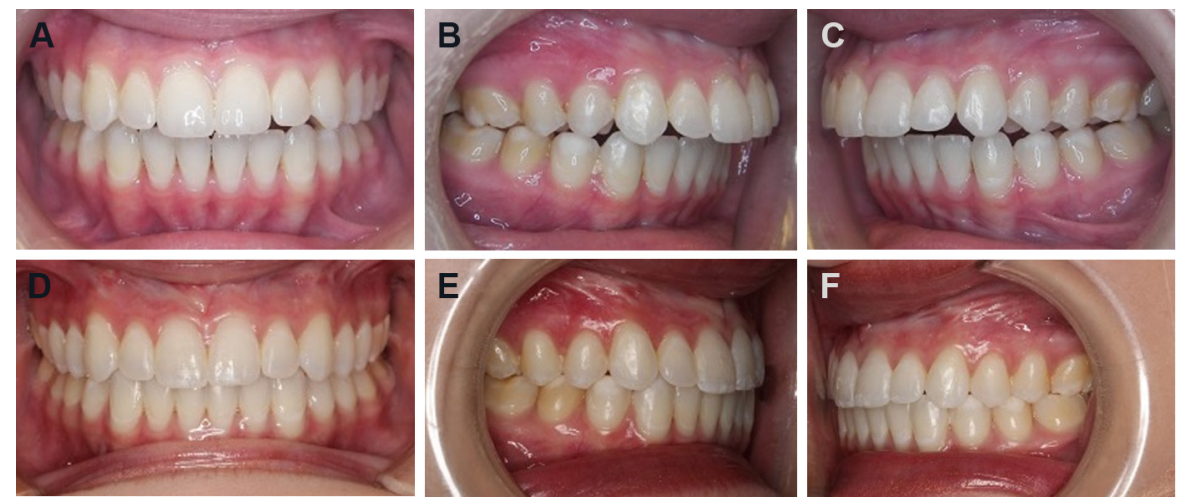

Figure 2. Case 1: Patient has a Class II malocclusion with an anterior open bite and occluding only on the molars. Frontal view (A). Right lateral view (B). Left lateral view (C). The occlusion is seen at 12 months post-surgery snowing a good occlusal outcome. Frontal view (D). Right lateral view (E). Left lateral view (F).

surgical protocol for teenage JIA patients with significant dentofacial deformities [Figure 1D-F, Figure 2DF, Figure 3B].

\section{Surgical protocol}

Based on a surgical prediction tracing [Figure 3B] developed from a presurgical lateral cephalometric radiograph analysis, and clinical evaluation, the desired final position of the maxilla and mandible is determined. The mandible is placed into the ideal functional and esthetic alignment with counterclockwise rotation (CCWR) using the patient-fitted TMJ Concepts TJP. The maxilla requires osteotomies for CCWR repositioning to maximize the functional and esthetic result. CT scans are taken extending from superiorposterior to the TMJ to anterior to the chin, maxilla and nasal bones adhering to the TMJ Concepts CT protocol. Virtual surgical planning (VSP) (3-D Systems, Littleton, CO) is used to perform the repositioning of the mandible and maxilla on a computer model, placing the jaws in the final planned position [Figure 7]. 

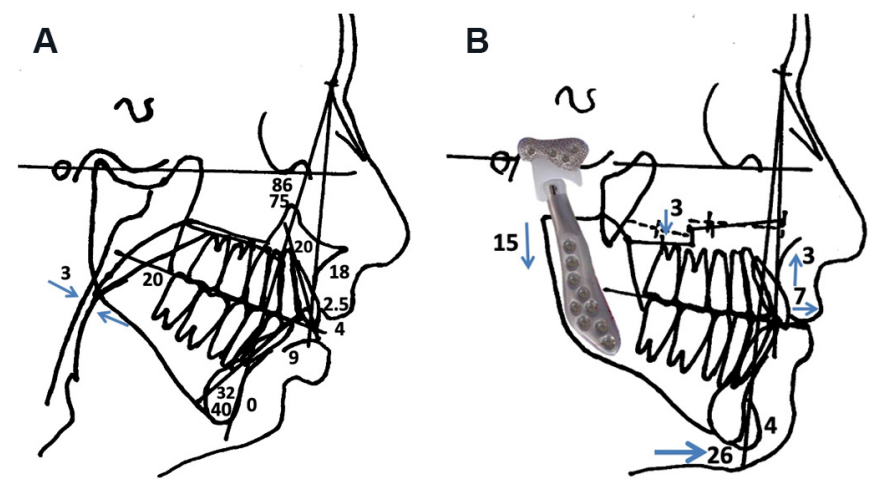

Figure 3. Case 1: Lateral cephalometric analysis demonstrating the retruded mandible and maxilla, with a high-occlusal plane angle facial morphology; common with the JIA TMJ disease process (A). The surgical prediction tracing demonstrates the skeletal movements achieved with TJP-Max-SP and genioplasty (B). JIA: Juvenile idiopathic arthritis; TMJ: temporomandibular joint; TJP-MaxSP: total joint prostheses with maxillary osteotomies surgical protocol.
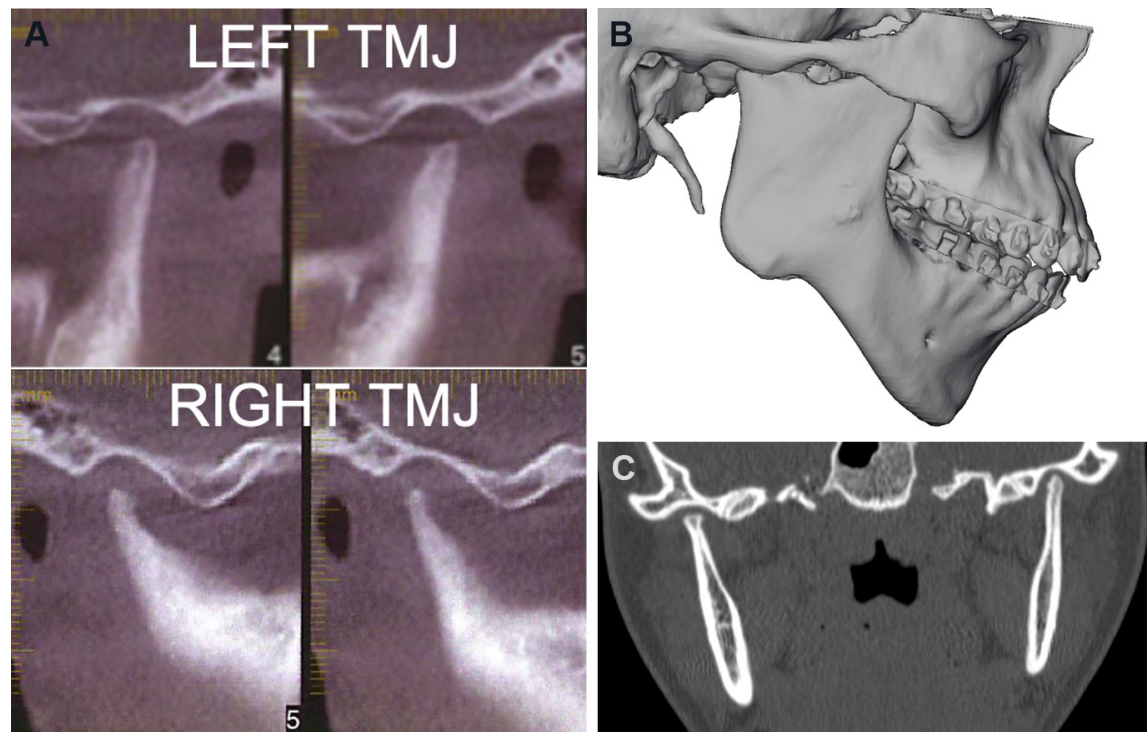

Figure 4. (A) CBCT scan sagittal views of the TMJs from Case 1, showing loss of condylar height and volume with narrow condylar stumps. (B) 3D CT scan, sagittal view demonstrating a wide condylar stump, more common with early age onset of JIA. Note the gonial notching and high occlusal plane angle skeletal morphology, with retruded mandible and maxilla. (C) Coronal view demonstrates significant narrowing of the condyles in the advanced stages of JIA. TMJs: Temporomandibular joints; JIA: juvenile idiopathic arthritis.

Any required recontouring of the ramus and fossa is usually done virtually. A 3D stereolithic model is produced with the mandible and maxilla in the final position and sent to TMJ Concepts for TJP manufacture [Figure 6]. The TJPs are then sent to the hospital for surgical implantation. Final dental models are obtained, maxillary dental model sectioned, equilibrations completed if indicated to maximize the occlusal fit, then sent to the VSP company for incorporation into the computer model for fabrication of the intermediate splint required to precisely reposition the mandible for prostheses placement, and final palatal surgical splint to stabilize the maxilla if segmented.

\section{Surgical sequencing}

The single-stage surgical protocol for the JIA patient with active or end-stage TMJ pathology includes the following: 

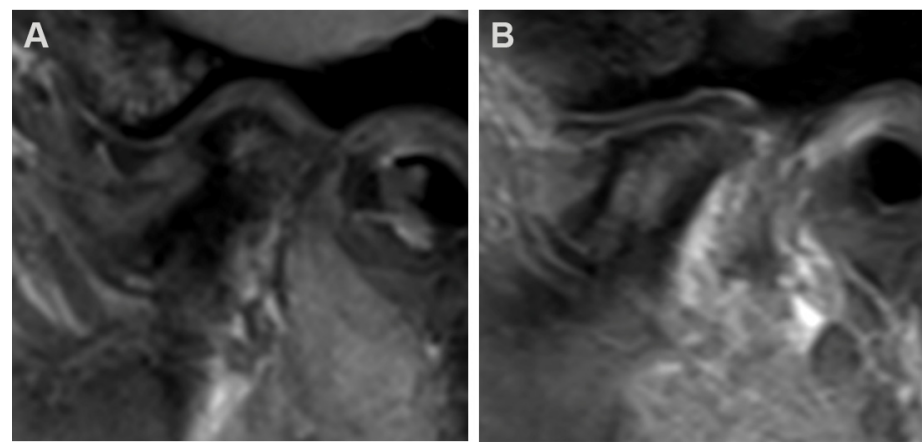

Figure 5. MRI of 15-year-old, JIA onset age 11 years, demonstrating condylar resorption and articular disc anteriorly displaced. The articular eminence has not been significantly resorbed (A). MRI of 16-year-old showing classic appearance with the articular disc in position over the resorbed condylar stump, but surrounded by a reactive pannus that causes resorption of the condyle and articular eminence as well as eventually destroys the articular disc (B). JIA: Juvenile idiopathic arthritis.

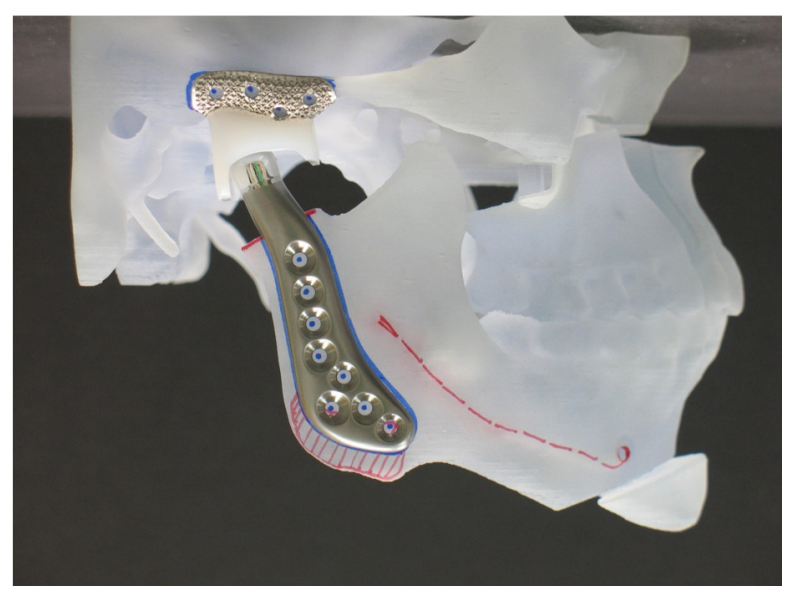

Figure 6. TMJ Concepts patient-fitted TJP positioned on the 3D stereolithic model with CCWR of the MMC, similar to Case 1. Note the posterior stop on the fossa component required to maximize the surgical outcome stability. TMJ: Temporomandibular joint; TJP: total joint prostheses; CCWR: counterclockwise rotation.

1. Surgical access for placement of the TJP through endaural and submandibular incisions.

2. Bilateral TMJ condylectomies (bone saved to graft maxilla).

3. Bilateral coronoidectomies (bone saved to graft maxilla).

4. Recontour fossa if indicated.

5. Detach the masseter and medial pterygoid muscles from the ramus.

6. Recontour ramus if indicated.

7. Mobilize the mandible.

8. Maxillo-mandibular fixation with intermediate surgical splint. 


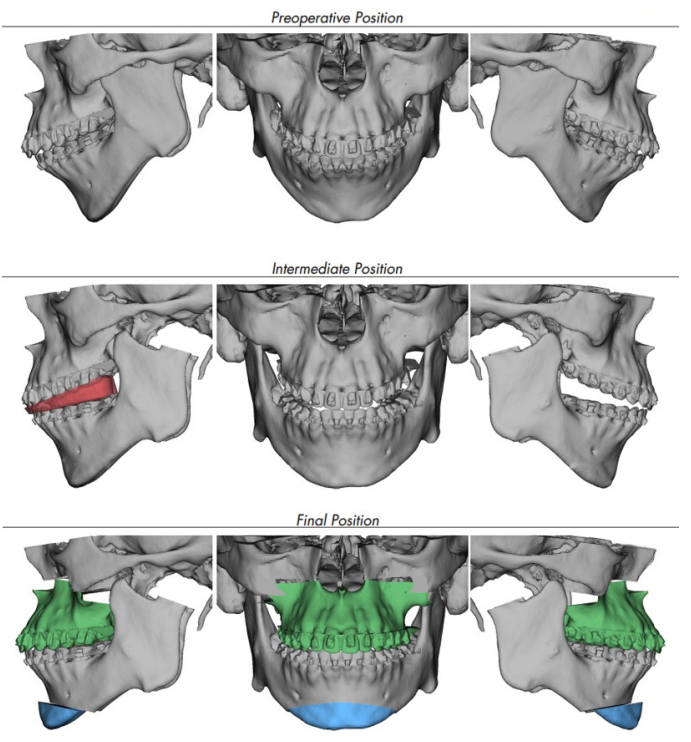

Figure 7. VSP of a patient similar to Case 1. The top row of images illustrates the presurgical jaw positions. Middle row of images shows the mandible CCWR and placed in its final position, while the maxilla remains in its original position. The intermediate splint is constructed to stabilize the mandible in its predetermined final position while the TMJ prostheses are inserted and secured. The bottom row shows the final position of the maxilla, mandible, and chin. CCWR: Counterclockwise rotation; TMJ: temporomandibular joint; VSP: virtual surgical planning.

9. Placement of total joint prostheses.

10. Masseter muscles reattached to mandible.

11. TMJ fat grafts packed around the prostheses, usually harvested from the abdomen.

12. Extra-oral incisions (endaural and submandibular) are closed.

13. Maxillary osteotomies and mobilization.

14. Turbinectomies, septoplasty, etc. if indicated.

15. Maxillary segmentation and application of the palatal splint if indicated.

16. Maxillary rigid fixation and bone grafting.

17. Adjunctive procedures such as genioplasty, rhinoplasty, facial augmentation, etc.

This TJP with maxillary osteotomy surgical protocol (TJP-Max-SP) used for the treatment of end-stage TMJ patients with dentofacial deformities was developed by Wolford in $1990^{[5,6]}$ and has remained unchanged, except for placement of fat grafts around the articulating area of the prostheses added to the protocol in $1992^{[16-18]}$. TJP-Max-SP provides highly predictable and stable outcomes for these patients including those with JIA [Figure 1D-F, Figure 2D-F] ${ }^{[5-14,19-21]}$. 
Post-surgery, no maxillo-mandibular fixation is used, but light inter-arch elastics are applied routinely to help support the mandible, since the muscles of mastication are reflected from the mandible to facilitate the CCWR and are initially non-functional. Postoperative elastics are generally discontinued following adequate functional return of the pterygo-masseteric musculature (usually 3-4 weeks post-surgery), unless elastics are required for finishing orthodontic mechanics. The palatal splint is usually removed 2 to 4 months postsurgery depending on the amount of maxillary expansion. Passive physical therapy begins approximately 2 weeks post-surgery to improve incisal opening and excursive movements. Patients are maintained on a soft diet for 4 months post-surgery to allow the maxilla to complete the initial bone-healing phase, and then patients are encouraged to progress toward a normal diet. Orthodontic appliances are usually maintained for a minimum of 4 to 6 months post-surgery to get through the initial healing phase, and then removed at the discretion of the orthodontist and patient placed in retainers. The healing process takes one year to complete $^{[22,23]}$.

\section{CASE REPORTS}

Case 1: A 16-year-old female was diagnosed with JIA at the age of eight years, involving most of her joints including her TMJs, creating a significant dentofacial deformity [Figure $1 \mathrm{~A}-\mathrm{C}$, Figure $2 \mathrm{~A}-\mathrm{C}$, Figure $3 \mathrm{~A}$ ]. She was treated with TJP-Max-SP and genioplasty [Figure 3B]. B-point advanced $18 \mathrm{~mm}$, pogonion $25 \mathrm{~mm}$, and occlusal plane decreased 14 degrees. At 12 months post-surgery, the patient exhibited good facial balance, occlusal and skeletal stability [Figure 1D-F, Figure 2D-F], MIO increased from $31 \mathrm{~mm}$ pre-surgery to 47 $\mathrm{mm}$ post-surgery, as well as significant improvement on the Likert scale for TMJ pain ( 8 to 0 ), headaches ( 3 to 0 ), jaw function ( 7 to 1 ), diet ( 2 to 0 ), disability ( 1 to 0 ), and significant improvement in quality of life.

Case 2: A 14-year-old female was diagnosed with JIA at the age of 6 years, involving most of her joints including her TMJs, creating a severe dentofacial deformity [Figure 8A-C, Figure 9A-C, Figure 10A]. She was treated with TJP-Max-SP and genioplasty [Figure 10B]. B-point advanced $26 \mathrm{~mm}$, pogonion $38 \mathrm{~mm}$, and occlusal plane decreased 27 degrees. At 21 months post-surgery, the patient exhibited good facial balance, occlusal and skeletal stability [Figure 8D-F, Figure 9D-F, Figure 10C], MIO increased from presurgery $43 \mathrm{~mm}$ to post-surgery $51 \mathrm{~mm}$, significant improvement in TMJ pain ( 7 to 0 ), headaches (8 to 0 ), jaw function ( 7 to 0 ), diet ( 7 to 0 ), disability ( 6 to 0 ), increase of oropharyngeal airway from $1 \mathrm{~mm}$ presurgery to $11 \mathrm{~mm}$ post-surgery, and significant improvement in quality of life.

\section{TJP-Max-SP outcome results}

JIA is a unique connective tissue disorder and with active or end-stage TMJ pathology, can create severe skeletal and occlusal deformities. The goals of surgical management are to: (1) establish normal TMJ and jaw function; (2) eliminate pain; (3) improve facial balance; (4) provide predictable skeletal and occlusal stability; and (5) establish a normal airway. As TMJ destruction worsens, clockwise rotation of the MMC occurs, leading to decreased masticatory and TMJ function along with worsening facial esthetics and exacerbation of sleep apnea. Frid et al. ${ }^{[2]}$ reviewed the literature for dentofacial deformities in JIA, comparing treatment modalities ranging from TMJ preservation, orthognathic surgery, distraction osteogenesis (DO), costochondral grafts (CCR), and alloplastic TJP. Based on the end-goal of improving patients' function and reducing TMJ pain, all treatment modalities were efficacious. However, compared to orthognathic surgery, DO, and CCR, TJP provided a single-stage procedure that addressed the functional and esthetic issues, and provided predictable stability, without requiring extended treatment time (DO), or associated donor site morbidity (CCR).

\section{Surgical effect on pain, function, and quality of life}

Henry and Wolford ${ }^{[25]}$ in 1993 reported on 107 patients that received autogenous tissue grafts or 

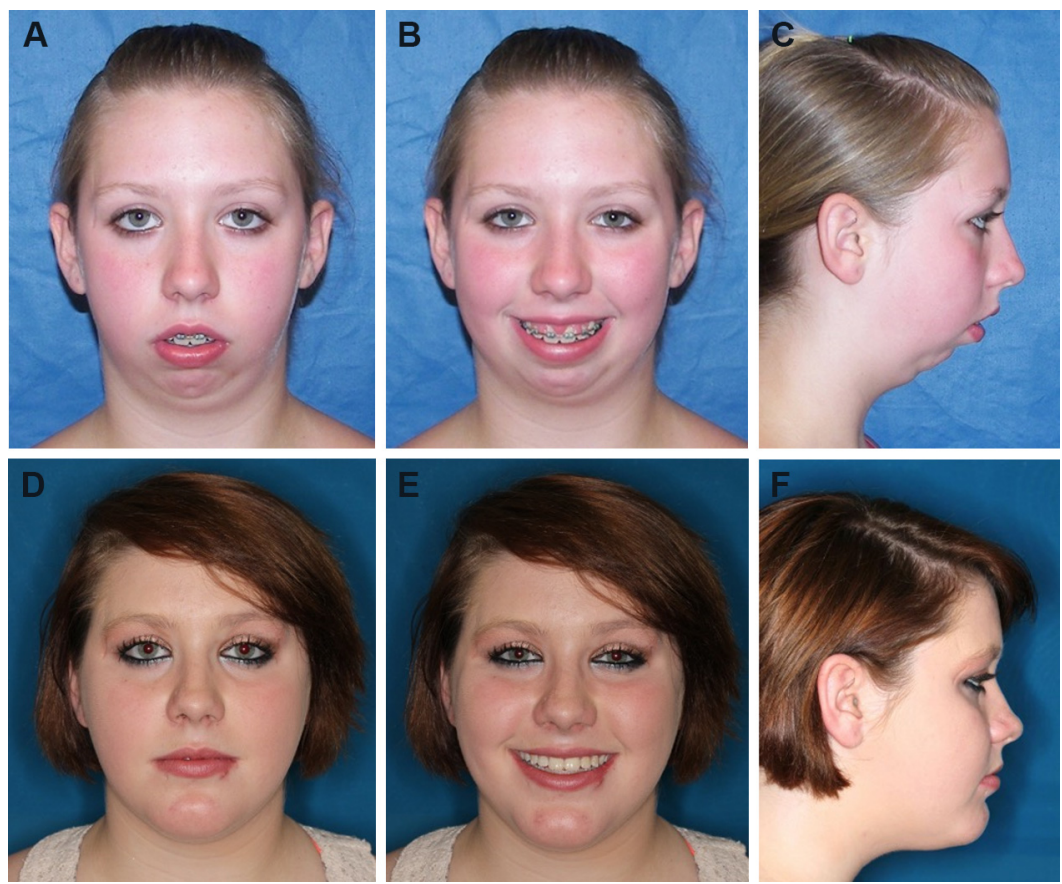

Figure 8. Case 2: 14-year-old female with JIA affecting the TMJs with major condylar resorption resulting in a severe dentofacial deformity. Frontal view (A). Smiling view (B). Profile view (C). The patient is seen 21 months post-surgery following TJP-Max-SP and genioplasty, resulting in significant improvement to the functional and esthetic result. Frontal view (D). Smiling view (E). Profile view (F). JIA: Juvenile idiopathic arthritis; TMJs: temporomandibular joints; TJP-Max-SP: total joint prostheses with maxillary osteotomies surgical protocol.
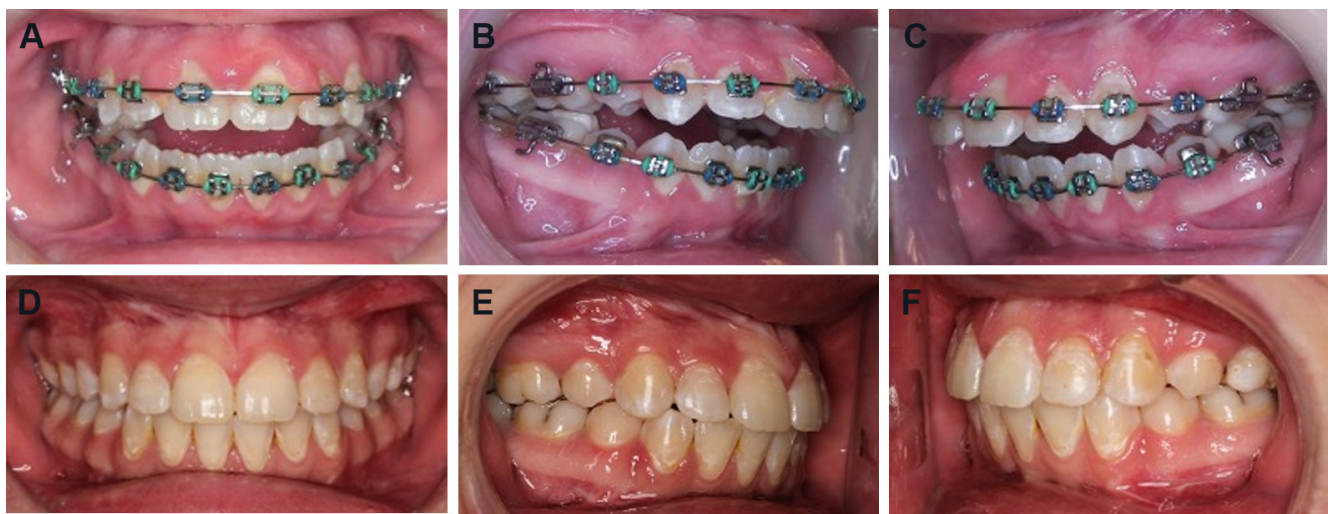

Figure 9. Case 2: Patient has a Class II malocclusion with an anterior open bite and occluding only on the molars. Frontal view (A). Right lateral view (B). Left lateral view (C). The occlusion is seen at 21 months post-surgery showing a good occlusal outcome. Frontal view (D). Right lateral view (E). Left lateral view (F).

Techmedica TJP to reconstruct TMJs severely damaged by failed Vitek Inc. Proplast/Teflon TMJ devices. A 4-year follow-up evaluation for pain, occlusal stability, and jaw function reported poor outcomes for autogenous tissue grafts with success ranging from only $8 \%$ to $31 \%$, while the Techmedica TMJ TJP had an $86 \%$ success, demonstrating the superiority of TJP over autogenous tissues in reconstructing the severely compromised TMJ. 

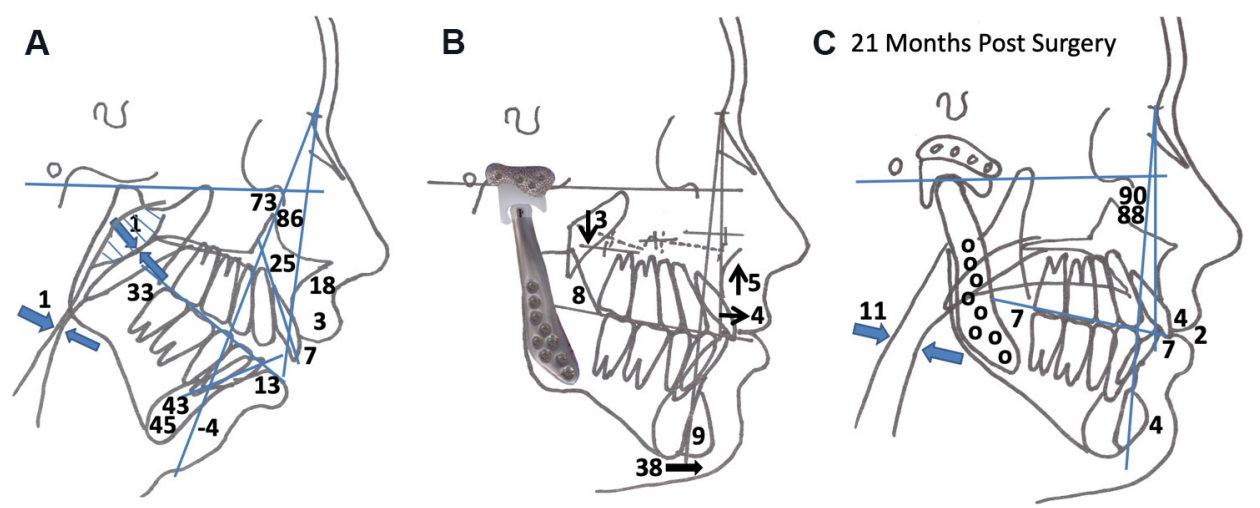

Figure 10. Case 2: (A) Lateral cephalometric analysis showing the high occlusal plane angle facial morphology including the significantly retruded mandible, anterior maxillary vertical hyperplasia, posterior maxillary hypoplasia, and severely decreased oropharyngeal airway. (B) Surgical prediction tracing shows TJP-Max-SP and genioplasty. (C) 21-month post-surgical cephalometric analysis shows good facial balance, and stability. Note the improvement of the oropharyngeal airway. TJP-Max-SP: Total joint prostheses with maxillary osteotomies surgical protocol.

Wolford et al. ${ }^{[5]}$ in 1994 reported on 56 patients (100 prostheses) receiving TMJ reconstruction with Techmedica TJP relative to pain, jaw function and occlusal stability. Patients with 0 to 1 previous TMJ surgery had success rates of $84 \%$ in the good outcome group, $16 \%$ in the fair group, and $0 \%$ in the poor group. For patients with 2 or more previous failed TMJ surgeries, the success rates decreased to $55 \%$ in the good group, $26 \%$ in the fair group, and $19 \%$ in the poor group, with pain as the major factor for poor results.

Pinto et al.${ }^{[17]}$ reported on 47 female patients with end-stage TMJ pathology treated with TJP-Max-SP. There was a statistically significant improvement at longest follow-up for TMJ pain, headaches, jaw function, diet, disability, and MIO.

Brownet al. ${ }^{[26]}$ reported improvement at longest follow-up (mean 30.9 months) with pain and dysfunction for 20 JIA patients treated with patient-fitted TJP, 11 of whom had concomitant maxillary osteotomies. Using a Likert scale, there were significant improvements in pain, headaches, diet, disability, and TMJ function. All patients at the time of surgery were in active treatment by a pediatric rheumatologist with 15 patients taking DMARDs prior to and at the time of surgery that was ineffective in controlling the condylar resorption, associated pain, and dysfunction.

Trivedi et al. ${ }^{[27]}$ evaluated 44 patients with TMJ JIA, treated with TJP-Max-SP. Mean age at surgery 20.4 years and follow-up 43 months. Using median Likert scores, TMJ pain improved from 4 to 0 , headaches 4 to 0 , jaw function 5 to 0 , diet 4 to 0 , and disability 4 to 0 . MIO improved from $36.4 \mathrm{~mm}$ to $43.7 \mathrm{~mm}$. Overall, $94.6 \%$ of the patients reported an improved quality of life, 2 remained the same, and none were worse.

\section{Surgical effect on skeletal and occlusal stability}

Dela Coleta et al. ${ }^{[16]}$ evaluated 47 female patients treated with TJP-Max-SP, mean follow-up 40.6 months. The mandible was advanced a mean of $18.4 \mathrm{~mm}$ at pogonion with relapse of $0.1 \mathrm{~mm}$, and the occlusal plane decreased a mean of 14.9 degrees with relapse of 0.6 degrees, documenting the stability of TJP-Max-SP.

Mehra et al ${ }^{[28]}$ evaluated 15 patients with rheumatoid arthritis, treated with TJP-Max-SP. Point B advanced a mean $21.7 \mathrm{~mm}$ with relapse $0.1 \mathrm{~mm}$, Pogonion advanced $29.2 \mathrm{~mm}$ with relapse $0.2 \mathrm{~mm}$, and the occlusal plane angle decreased $20.7^{\circ}$ with relapse $0.4^{\circ}$. This study demonstrated the surgical stability of TJP-Max-SP in the rheumatoid patient. 
Mehra et al. ${ }^{[29]}$ evaluated outcomes for patients with autoimmune or connective tissue diseases who had TMJ reconstruction divided into 2 groups. Group $1(n=9)$ were reconstructed with either rib grafts or sternoclavicular grafts, and for Group $2(n=20), 13$ were reconstructed with TJP-Max-SP and 7 with only TJP. Group 1 relapsed 32\% at Point B, whereas Group 2 relapsed 5\% at Point B, indicating significantly better stability with the TJP-Max-SP or only TJP compared to autogenous grafts.

Wolford and Kesterke ${ }^{[30]}$ reported outcomes for 42 JIA patients treated with TJP-Max-SP. Mean age at surgery 21.6 years and follow-up was 44.2 months. Mean advancement at Point B was $15.9 \mathrm{~mm}$, pogonion $23.8 \mathrm{~mm}$, and occlusal plane decreased $17.2^{\circ}$. Long-term post-surgery, there were no statistically significant changes for any of these anatomical landmarks. All devices were well tolerated by the patients and none of the devices required removal.

\section{Surgical effect on airway}

Kortebein and Wolford ${ }^{[31]}$ were the first to demonstrate the positive effects of CCWR of the MMC on the post-surgical improvement of the oropharyngeal airway. Reiche-Fischel et al. ${ }^{[32]}$ evaluated 72 patients relative to the amount of mandibular advancement and effect on the increase in the oropharyngeal airway measured at the tongue base. Advancement from 0 to $10 \mathrm{~mm}$ had a $66 \%$ increase of the oropharyngeal airway, that from 10 to $15 \mathrm{~mm}$ had an increase of $56 \%$, and for that $>20 \mathrm{~mm}$, the increase was $41 \%$, indicating the greatest change in the airway dimension occurs with the first $10 \mathrm{~mm}$ of CCWR of the MMC.

Mehra et al.$^{[33]}$ evaluated 30 patients with CCWR of the MMC where the mandibular advancement averaged $7.5 \mathrm{~mm}$. The oropharyngeal airway space increased $76 \%$ of the amount of mandibular advancement at the base of the tongue and $47 \%$ at the soft palate level.

Goncalves et al. ${ }^{[34]}$ evaluated 56 patients and showed an average mandibular advancement of $13.1 \mathrm{~mm}$ with the CCWR of the MMC with the airway measurement increased at the retroglossal area $4.4 \mathrm{~mm}$ and remained stable for a long term.

Coleta et al. ${ }^{[35]}$ reported on 47 females treated with TJP-Max-SP. Mandibular advancement at menton was $17.3 \mathrm{~mm}$. The oropharyngeal airway at the narrowest retroglossal airway space pre-surgery was $7.3 \mathrm{~mm}$ and post-surgery increased an additional $4.9 \mathrm{~mm}$ to $12.2 \mathrm{~mm}$ (67\% increase).

Gonçalves et al. ${ }^{[36]}$ performed a 3-dimensional assessment of airway changes following TJP-Max-SP. The average mandibular advancement was $9.65 \mathrm{~mm}$ at point $\mathrm{B}$. The AP dimension of the retroglossal area presurgery was $7.9 \mathrm{~mm}$ and increased by $3 \mathrm{~mm}$. The oropharyngeal surface area increased by $42 \%$ and the oropharyngeal airway volume increased by $72 \%$.

Yuen et al. ${ }^{[37]}$ reported on 137 patients receiving TJP-Max-SP. Pre-surgery, the oropharyngeal volume mean was $8.97 \mathrm{~cm}^{3}$ and post-surgery increased to $15 \mathrm{~cm}^{3}$ for a $67 \%$ volume increase. A cross-sectional area at the soft palate level increased post-surgery by $95 \%$ and a cross-sectional at the base of the tongue increased by $57 \%$.

Wolford and Kesterke ${ }^{[30]}$ in 2021 , reported on oropharyngeal airway surgical outcomes for 42 JIA patients treated with TJP-Max-SP. The mean age at surgery was 21.6 years and follow-up 44.2 months. Mean advancement at Point B $16 \mathrm{~mm}$, pogonion $24 \mathrm{~mm}$, and occlusal plane decreased $17^{\circ}$. The mean oropharyngeal airway dimension pre-surgery was $4 \mathrm{~mm}$ at the soft palate and increased to $9 \mathrm{~mm}(125 \%$ increase), and at the tongue base pre-surgery was $4 \mathrm{~mm}$ and increased to $10 \mathrm{~mm}$ ( $150 \%$ increase). 
Although the oropharyngeal airway is not a true cylinder, according to Poiseuille's law and equation, doubling the diameter of a cylinder increases the airflow 4 -fold ${ }^{[38,39]}$. The oropharyngeal airway at the soft palate and tongue base levels increased by more than double the original diameter, so airflow should be significantly improved.

\section{Age for surgical intervention}

Predictability of results and limiting correction of the jaw and TMJ related deformities to one major operation can best be achieved by waiting until growth is relatively complete. Females usually have the majority of their facial growth (98\%) complete by 15 years and males by the age of 17 to 18 years ${ }^{[40]}$. Performing TMJ TJP and orthognathic surgery during earlier growth years presents a risk of requiring additional surgery at a later time to correct antero-posterior discrepancy and malocclusion that can develop during completion of growth. There are definite indications for early surgery in the JIA patient such as uncontrollable pain, severe jaw deformity, difficulties eating and chewing, severe airway compromise (sleep apnea), psychosocial instability, etc. Wolford et al. ${ }^{[41-44]}$ have previously published on maxillary and mandibular orthognathic surgery as well as TMJ pathological conditions and surgery relative to the adverse effects on growth, with age consideration guidelines. Younger patients will have a greater adverse effect on jaw growth with surgery with a higher probability of requiring repeat surgery following completion of growth. If repeated orthognathic surgery is required, the advancement of the mandible can be accomplished by one of these surgical options: (1) intraoral ramus sagittal split osteotomy; (2) extraoral sagittal split ramus osteotomy; (3) advance the mandible forward relative to the prosthesis by removing the screws from the mandibular component, separate it from the ramus, advance the mandible, and re-fixate the prosthesis with bone screws to the mandible in its new position; or (4) replace the mandibular components of the TJP with a new longer mandibular component that would be reattached to the mandibular ramus after the mandible is moved forward to its new position. A repeat of the maxillary osteotomies will also be required. The author prefers to perform surgery on JIA females at the age of 14 years or older and males 16 years or older to provide predictable results without requiring additional surgery.

Although the life expectancy of the TMJ Concepts TJP is unknown, Wolford et al. ${ }^{[18]}$ published a 20-year follow-up study of 56 patients who had received the Techmedica TJP between 1989 and 1993. There were statistically significant improvements for MIO, jaw function, TMJ pain, and diet, with $85.7 \%$ of the patients reporting significant improvement in their quality of life. There were no reports of device removal due to material wear or failure.

\section{SUMMARY}

JIA patients with TMJ involvement, resultant jaw deformity, malocclusion, with or without pain and airway compromise, may benefit from surgical intervention. TMJ Concepts patient-fitted TJP for TMJ and mandibular reconstruction in conjunction with maxillary orthognathic surgery for counterclockwise rotation-advancement of the maxillo-mandibular complex provides improvement relative to TMJ pain, headaches, jaw function, diet, disability, airway, incisal opening, long-term occlusal and skeletal stability, as well as quality of life.

\section{DECLARATIONS}

\section{Authors' contributions}

The author contributed solely to the article. 


\section{Availability of data and materials}

Not applicable.

\section{Financial support and sponsorship}

None.

\section{Conflicts of interest}

The author declared that there are no conflicts of interest. Neither Dr. Wolford or any members of his family have a financial arrangement or affiliation with any corporations, commercial products, or services that are discussed in this document.

\section{Ethical approval and consent to participate}

Not applicable.

\section{Consent for publication}

The author declares that signed Informed Consents were obtained for publication of patients' images used in this manuscript.

\section{Copyright}

(c) The Author(s) 2021.

\section{REFERENCES}

1. Ravelli A, Martini A. Juvenile idiopathic arthritis. Lancet 2007;369:767-78. DOI PubMed

2. Petty RE, Southwood TR, Manners P, et al. International league of associations for rheumatology classification of juvenile idiopathic arthritis: second revision, Edmonton, 2001. J Rheumatol 2004;31:390-2. PubMed

3. Still GF. On a form of chronic joint disease in children. Med Chir Trans 1897;80:47-59. PubMed PMC

4. Ronchezel MV, Hilario MO, Goldenberg J, et al. Temporomandibular joint and mandibular growth alterations in patients with juvenile rheumatoid arthritis. J Rheumatol 1995;22:1956-61. PubMed

5. Wolford LM, Cottrell DA, Henry CH. Temporomandibular joint reconstruction of the complex patient with the techmedica custommade total joint prosthesis. J Oral Maxillofac Surg 1994;52:2-10. DOI PubMed

6. Wolford LM, Pinto LP, Cárdenas LE, Molina OR. Outcomes of treatment with custom-made temporomandibular joint total joint prostheses and maxillomandibular counter-clockwise rotation. Proc (Bayl Univ Med Cent) 2008;21:18-24. DOI PubMed PMC

7. Wolford LM, Dhameja A. Planning for combined TMJ arthroplasty and orthognathic surgery. Atlas Oral Maxillofac Surg Clin North Am 2011;19:243-70. DOI PubMed

8. Movahed R, Teschke M, Wolford LM. Protocol for concomitant temporomandibular joint custom-fitted total joint reconstruction and orthognathic surgery utilizing computer-assisted surgical simulation. J Oral Maxillofac Surg 2013;71:2123-9. DOI PubMed

9. Wolford LM, Movahed R. Concomitant TMJ and Orthognathic Surgery: diagnosis and treatment planning. Oral and Maxillofacial Surgery Knowledge Update. AAOMS 2014.

10. Wolford LM, Movahed R. Combined TMJ and orthognathic surgery. In: Kademani D, Tiwana P, editor. Atlas of Oral and Maxillofacial Surgery. Elsevier Saunders; 2016. p. 1364-77.

11. Wolford LM. Concomitant TMJ total joint replacement and orthognathic surgery. In: Mercuri LG, editor. Temporomandibular Joint total joint replacement-TMJ TJR. Switzerland: Springer International Publishing; 2016. p 133-64.

12. Wolford LM. Concomitant TMJ total joint prosthetic reconstruction and orthognathic surgery. In: Turvey TA, Costello BJ, Ruiz RL, editors. Fonseca Oral and Maxillofacial Surgery. St. Louis: Elsevier; 2018. p. 222-45.

13. Wolford LM, Mehra P. Custom-made total joint prostheses for temporomandibular joint reconstruction. Proc (Bayl Univ Med Cent) 2000;13:135-8. DOI PubMed PMC

14. Wolford LM, Pitta MC, Reiche-Fischel O, Franco PF. TMJ concepts/techmedica custom-made TMJ total joint prosthesis: 5-year follow-up study. Int J Oral Maxillofac Surg 2003;32:268-74. DOI PubMed

15. Mercuri LG, Wolford LM, Sanders B, White R, Hurder A, Henderson W. Custom CAD/CAM total temporomandibular joint reconstruction system: preliminary multicenter report. J Oral Maxillofac Surg 1995;53:106-15. DOI PubMed

16. Wolford LM, Karras SC. Autologous fat transplantation around temporomandibular joint total joint prostheses: Preliminary treatment outcomes. J Oral Maxillofac Surg 1997;55:245-51. DOI PubMed

17. Wolford LM, Morales-Ryan CA, Morales PG, Cassano DS. Autologous fat grafts placed around temporomandibular joint total joint prostheses to prevent heterotopic bone formation. Proc (Bayl Univ Med Cent) 2008;21:248-54. DOI PubMed PMC

18. Wolford LM, Cassano DS. Autologous fat grafts around temporomandibular joint (TMJ) total joint prostheses to prevent heterotopic bone. In: Shiffman MA, editor. Autologous fat transfer. Berlin: Springer; 2010. p. 361-82. 
19. Coleta KE, Wolford LM, Gonçalves JR, Pinto Ados S, Pinto LP, Cassano DS. Maxillo-mandibular counter-clockwise rotation and mandibular advancement with TMJ Concepts total joint prostheses: part I--skeletal and dental stability. Int J Oral Maxillofac Surg 2009;38:126-38. DOI PubMed

20. Pinto LP, Wolford LM, Buschang PH, Bernardi FH, Gonçalves JR, Cassano DS. Maxillo-mandibular counter-clockwise rotation and mandibular advancement with TMJ Concepts total joint prostheses: part III--pain and dysfunction outcomes. Int J Oral Maxillofac Surg 2009;38:326-31. DOI PubMed

21. Wolford LM, Mercuri LG, Schneiderman ED, Movahed R, Allen W. Twenty-year follow-up study on a patient-fitted temporomandibular joint prosthesis: the techmedica/TMJ concepts device. J Oral Maxillofac Surg 2015;73:952-60. DOI PubMed

22. Wolford LM. Postsurgical patient management. In: Fonseca RJ, Marciani RD, Turvey TA, editors. Oral and maxillofacial surgery. St. Louis: Saunders Elsevier; 2008. p. 396-418.

23. Wolford LM, Rodrigues DB, Limoeiro E. Orthognathic and TMJ surgery: postsurgical patient management. J Oral Maxillofac Surg 2011;69:2893-903. DOI PubMed

24. Frid P, Resnick C, Abramowicz S, Stoustrup P, Nørholt SE; Temporomandibular joint juvenile arthritis work group TMJaw. Surgical correction of dentofacial deformities in juvenile idiopathic arthritis: a systematic literature review. Int J Oral Maxillofac Surg 2019;48:1032-42. DOI PubMed

25. Henry CH, Wolford LM. Treatment outcomes for temporomandibular joint reconstruction after Proplast-Teflon implant failure. J Oral Maxillofac Surg 1993;51:352-8. DOI PubMed

26. Brown Z, Rushing DC, Perez DE. Alloplastic temporomandibular joint reconstruction for patients with juvenile idiopathic arthritis. $J$ Oral Maxillofac Surg 2020;78:1492-8. DOI PubMed

27. Trivedi B, Wolford LM, Kesterke M. Can combined temporomandibular joint reconstruction with patient-fitted total joint prostheses and orthognathic surgery reduce symptoms in juvenile idiopathic arthritis patients? J Oral Maxillofacial Surg. Forthcoming 2021.

28. Mehra P, Wolford LM, Baran S, Cassano DS. Single-stage comprehensive surgical treatment of the rheumatoid arthritis temporomandibular joint patient, J Oral Maxillofac Surg 2009;67:1859-72. DOI PubMed

29. Mehra P, Henry CH, Giglou KR. Temporomandibular joint reconstruction in patients with autoimmune/connective tissue disease. $J$ Oral Maxillofac Surg 2018;76:1660-4. DOI PubMed

30. Wolford LW, Kesterke M. Can combined temporomandibular joint reconstruction with patient fitted total joint prosthesis and orthognathic surgery provide stable skeletal and occlusal outcomes in juvenile idiopathic arthritis patients? J Oral Maxillofacial Surg Forthcoming 2021 .

31. Kortebein MJ, Wolford LW. The effect of maxillary and mandibular advancement with decrease of occlusal plane on the posterior airway space. J Oral Maxillofac Surg 1991;49:93. DOI

32. Reiche-Fischel O, Wolford LM, Pitta MC, Kortebein MJ, Franco PF. Posterior airway changes after double jaw surgery with counterclockwise rotation. J Oral Maxillofac Surg 1996;54:96.

33. Mehra P, Downie M, Pita MC, Wolford LM. Pharyngeal airway space changes after counterclockwise rotation of the maxillomandibular complex. Am J Orthod Dentofacial Orthop 2001;120:154-9. DOI PubMed

34. Goncalves JR, Buschang PH, Goncalves DG, Wolford LM. Postsurgical stability of oropharyngeal airway changes following counterclockwise maxillo-mandibular advancement surgery. J Oral Maxillofac Surg 2006;64:755-62. DOI PubMed

35. Coleta KE, Wolford LM, Gonçalves JR, Pinto Ados S, Cassano DS, Gonçalves DA. Maxillo-mandibular counter-clockwise rotation and mandibular advancement with TMJ concepts total joint prostheses: part II-airway changes and stability. Int J Oral Maxillofac Surg 2009;38:228-35. DOI PubMed

36. Gonçalves JR, Gomes LC, Vianna AP, Rodrigues DB, Gonçalves DA, Wolford LM. Airway space changes after maxillomandibular counterclockwise rotation and mandibular advancement with TMJ Concepts ${ }^{\circledR}$ total joint prostheses: three-dimensional assessment. Int J Oral Maxillofac Surg 2013;42:1014-22. DOI PubMed

37. Yuen H, Rossouw PE, Wolford LM, Wang H. Pharyngeal airway space changes after condylar replacement and mandibular advancement surgery. J Oral Maxillofac Surg 2018;76:1165-74. DOI PubMed

38. Sutera SP, Skalak R. The history of Poiseuille's law. Annu Rev Fluid Mech 1993;25:1-19. DOI

39. Pfitzner J. Poiseuille and his law. Anaesthesia 1976;31:273-5. DOI PubMed

40. Riolo ML, Moyers RE, McNamara JA, Hunter WS. An atlas of craniofacial growth. cephalometric standards from the university school growth study, the University of Michigan. Ann Arbor: Center for Human Growth and Development, University of Michigan;1974. p. 105-6.

41. Wolford LM, Karras SC, Mehra P. Considerations for orthognathic surgery during growth, part 1: mandibular deformities. Am J Orthod Dentofacial Orthop 2001;119:95-101. DOI PubMed

42. Wolford LM, Karras SC, Mehra P. Considerations for orthognathic surgery during growth, part 2: maxillary deformities. Am J Orthod Dentofacial Orthop 2001;119:102-5. DOI PubMed

43. Wolford LM, Rodrigues DB. Orthognathic considerations in the young patient and effects on facial growth. In: Preedy VR, editor. Handbook of growth and growth monitoring in health and disease. New York: Springer; 2012. p. 1789-808.

44. Wolford LM, Rodrigues DB. Temporomandibular joint (TMJ) pathologies in growing patients: effects on facial growth and development. In: Preedy VR, editor. Handbook of growth and growth monitoring in health and disease. New York: Springer; 2012. p. 1809-28. 